\title{
Modeling language learning using specialized Elo ratings
}

\author{
${ }^{\dagger}$ Jue Hou, ${ }^{\dagger}$ Maximilian W. Koppatz, ${ }^{\dagger}$ José María Hoya Quecedo, \\ ${ }^{\star}$ Nataliya Stoyanova, ${ }^{\ddagger}$ Mikhail Kopotev and ${ }^{\dagger}$ Roman Yangarber \\ †University of Helsinki, Department of Computer Science, Finland \\ ${ }_{\ddagger}^{\ddagger}$ University of Helsinki, Department of Modern Languages, Finland \\ first.last@helsinki.fi \\ ${ }^{\star}$ University of Milan, Department of Foreign Languages and Literatures, Italy \\ first.lasteunimi.it
}

\begin{abstract}
Automatic assessment of the proficiency levels of the learner is a critical part of Intelligent Tutoring Systems. We present methods for assessment in the context of language learning. We use a specialized Elo formula used in conjunction with educational data mining. We simultaneously obtain ratings for the proficiency of the learners and for the difficulty of the linguistic concepts that the learners are trying to master. From the same data we also learn a graph structure representing a domain model capturing the relations among the concepts. This application of Elo provides ratings for learners and concepts which correlate well with subjective proficiency levels of the learners and difficulty levels of the concepts.
\end{abstract}

\section{Introduction}

A key goal of Intelligent Tutoring Systems (ITS) is to provide students with individualized learning, and thus support their learning process. Several systems for various subjects have proven to be effective, (Ritter et al., 2007; Arroyo et al., 2014; Klinkenberg et al., 2011). Our work is part of an international collaborative effort to developing large-scale Intelligent Computer-Aided Language Learning (ICALL) systems for use in real-world environments. Our system, Revita (Katinskaia et al., 2018), is in use in official university-level curricula at several major universities, to enhance language learning and teaching. ${ }^{1}$

To develop automated methods for personalized tutoring, robust models for assessing the current proficiency of learners are required. Such models enable quantitative evaluation and comparison of teaching methods with respect to the rate of development of the learners, which enables us to evaluate the performance of the ICALL system.

\footnotetext{
${ }^{1}$ https://revita.cs.helsinki.fi/
}

Several approaches for modeling knowledge have been proposed, such as Bayesian Knowledge Tracing (Corbett and Anderson, 1994), Learning Factor Analysis (Cen et al., 2006), and its more advanced variant-Performance Factor Analysis, (Pavlik Jr et al., 2009). These models, however, are complex and time-consuming to implement for ITS, and require large amounts of data for each learner, (Pelánek, 2016).

In this work, we present a simple and effective method for assessing the proficiency of language learners, as well as the difficulty of linguistic concepts, by utilizing the Elo formula, (Elo, 1978)in an unsupervised fashion. The result is a set of rated concepts and a method for assessing the current and historical proficiency of each learner. We also present a concept graph-learned from the educational data-representing the dependencies between concepts.

We use educational data, collected from real language learners, in two assessment contexts. One context is where learners take language proficiency tests. During the test the system samples questions from a database, each question linked to a specific linguistic concept. Examples of concepts are collocations, certain type of verb inflection, certain word-order rules, etc. In the second context, learners do exercises based on "authentic" texts-chosen based on the learners' intereststhat have a difficulty estimate, assigned by a statistical model. In each context, the result is a numeric Elo-based rating describing learners' proficiency in the target language.

The paper is organized as follows. Section 2 outlines out data generation process. In Section 3, we describe the Elo rating system, and discuss our modifications to the formulas in the two assessment contexts. In Section 4, we discuss our approach for building a domain model, represented by a concept graph. Section 5 describes simula- 
tions and experiments on obtaining Elo ratings for concepts and for assessing learner competency. In Section 6, we discuss preliminary results on correlation between Elo ratings in different assessment contexts, and the correlation between the Elo ratings and the performance levels assigned by teachers. We also demonstrate a graph of concepts, each with Elo difficulty estimates. In Section 7, we discuss current problems. Section 8 concludes with current directions of research.

\section{Data}

This work builds on educational data we have collected through a collaborative effort with language teachers at several universities. In this paper, we focus on students learning Russian as a second language (L2) at different levels on the CEFR scale, ranging from A1 to C2, (Little, 2007). The students yield data in two assessment contexts:

Language tests: In the testing context, the students take online language tests on a platform provided by the system. Each test is time-limited, and comprised of approximately 300 test items, sampled from a database of 3390 multiple-choice questions. The questions were prepared by language teachers and linguistic experts over a period of 20 years, (Kopotev, 2012, 2010). Each question is linked to one of 140 linguistic "concepts," also predefined by the experts. At the time of this writing, the response data consists of 600000 test answers, by approximately 1000 learners. For each answer we record to which concept the question belongs, whether the answer was correct, as well as timestamps.

Language practice: In the practice context, students practice the language by doing exercises based on a text. The response data consists of student answers to the exercises: one set of exercises is associated with a snippet of text (e.g, one paragraph). The system offers various types of exercises, including multiple-choice questions, "cloze" quizzes (fill-in-the-blank), and listening comprehension, which are generated automatically based on the text chosen by the learner. Each text has been assigned a difficulty rating by a linear model, described in 3.4. Currently, we do not use information about to which linguistic concepts each exercise belong. For each attempted set of exercises, we use the percentage of correct answers, text difficulty, and the timestamp.

\section{Rating methods}

This section describes how we calculate and update ratings of users and linguistic concepts. We cover the two assessment contexts in which the data were generated: first, the testing context, and second, the practice context. The estimator of text difficulty is described at the end of this section.

\subsection{Elo ratings}

The Elo rating, introduced by Arpad Elo, (Elo, 1978), was originally used for rating the skills of chess players, and evolved versions of it are now widely used in a variety of domains, ranging from video-games to Tinder.

The Elo formula defines the expected result of actor $A$ in a match against actor $B$ according to:

$$
E_{A}=\frac{1}{1+10^{\frac{R_{B}-R_{A}}{\sigma}}}
$$

$E_{A}$ is a value between 0 and 1 , indicating the expectation (probability) of success/win. $R_{X}$ refers to the current rating of actor $X$, and $\sigma$ is a scale variable. The scale is traditionally set to 400 (in chess). This controls the spread of the resulting ratings. In the present work, we used $\sigma=600 .^{2}$

The rating of actor $A$ after a match with another actor is completed is updated according to:

$$
R_{A}^{\prime}=R_{A}+K\left(S_{A}-E_{A}\right)
$$

The factor $K$ controls the maximum rating adjustment that is possible at one time. We use a static $K$ value of $32 . S_{A}$ denotes the outcome, or the score of a match for actor $A$ : loss, draw and win for $A$ is denoted as $0,0.5$ and 1 , respectively.

The Elo rating method has three important properties. First, the formulas are symmetric: $A$ and $B$ switch places when calculating with respect to $B$. This leads to the zero-sum property of the Elo rating distribution, when $K$ is static - the amount of Elo lost by the losing actor is transferred to the winning one. As a result, the mean of the ratings of all actors will be whatever the initial rating is set to. We initially set the rating of all players (concepts and learners) to 1500 .

Second, the magnitude of the rating update depends on the difference between the outcome $S_{A}$

\footnotetext{
${ }^{2}$ The reason for this is largely aesthetic; the number of users is currently small (about 1000), which results in a somewhat narrow spread of the rating distribution. A larger spread is more comparable to actual chess ratings, which is more familiar and easier to conceptualize.
} 
and the expected score $E_{A}$. This means that a highly rated actor failing against a significantly lower rated one will have a severe loss in Elo, approaching the value of $K$. Conversely, the success of a higher rated actor is expected, thus it yields a small update in the rating. If the actors are evenly matched the update is between the two extremes.

Finally, the final, or "current," distribution of Elo ratings in a system depends on the order of the matches. This implies that the Elo rating is a representation of an actor's current proficiency. Consider an actor who fails in the first half of 100 matches, and succeeds in the second half, and compare the result to the reverse order. Because the rating updates decrease toward the expected extremes, and are exaggerated at the unexpected extremes, the resulting rating of the actor will be high when his successes are in the second half of the matches and low in the reverse case-even though the two sequences are events are the same, and differ only in their ordering.

\subsection{Language test ratings}

In (Klinkenberg et al., 2011; Pelánek, 2016), it was shown that Elo ratings can be adapted for use in educational systems, to model the proficiency of students and the difficulty of questions. In our system, in the testing context, the analogue of an outcome of a "game" is a student attempting an exercise; the two rated "actors" are the student and the exercise. $S_{A}$ represents whether a student has answered the question correctly. The rating $R_{Q}$ of a question $Q$ captures the difficulty of the question.

An adjustment is made for multiple-choice problems to account for the fact that students have some chance of guessing correctly, even if they do not know the correct answer. For this, we adopt an approach recommended by Pelánek (2016), penalizing the expected value by the probability that a random guess is correct. A similar expectation formula is proposed in Item Response Theory (Embretson and Reise, 2013).

In (Klinkenberg et al., 2011; Pelánek, 2016), the focus was assessing the difficulty of each particular question. In our system, we model the concept category to which the questions belong; every question from a concept is a representative for the concept in the calculations. In our data, each question is linked to one linguistic concept. The formula for the expectation of student $S$ having a correct response on a question from Concept $C$ adjusted for guessing is:

$$
E_{A}=\frac{1}{k} \cdot 1+\left(1-\frac{1}{k}\right) \cdot \frac{1}{1+10^{\frac{R_{C}-R_{S}}{\sigma}}},
$$

where $k$ is the number of choices in the multiplechoice question. We expect the Elo ratings for concepts to approach their true value after a large number of data points ("games" or exercise attempts) have been sampled. To obtain concept ratings of better quality, they are learned by re-adjusting all ratings by re-playing all games/attempts in chronological order over several epochs. We call this the Elo "burn-in" period, and describe it in Section 5.1. This is used to obtain "stable" concept ratings.

\subsection{Language practice ratings}

In the practice context, the exercises are different from the test context. Our method therefore differs in the two contexts in several ways. Here, the exercises sets that the learners have answered are composed of a variety of exercises, some of which may not be linked to a specific linguistic concept (recall, each test item is linked to a concept). This means we cannot share the ratings directly between the two learning contexts.

To address this problem, we make the simplifying assumption that on average the exercises in a given text correspond to the difficulty rating of the text. The method for estimating the difficulty of a text is described in the following subsection. ${ }^{3}$

Concretely, we define $S_{A}$ as the percentage of correct responses in a given set of exercises. $E_{A}$ for a set of exercises is set to the Elo rating of the entire text from which the exercises are drawn.

We update the learner's Elo after each set of exercises, but update the text's Elo only after an entire iteration through the text. Crucially, the system updates the Elo for the text only with respect to the specific user who practiced it. This models the notion that as I practice with the same text over and over, the text becomes "easier" for mebut not for other players.

\subsection{Estimator of text difficulty}

Modeling and characterizing the readability of texts is a well-studied problem with a long history, (Dubay, 2009). Experiments with lexical and

\footnotetext{
${ }^{3}$ This means that A. our difficulty model should return an accurate estimate of the complexity of the text, and B. that these difficulty estimates should be properly calibrated to the desired rating scale.
} 
grammatical features have been conducted, (Chen and Meurers, 2016; Heilman et al., 2008).

We use a simple linear model for estimating the difficulty of a given text. The output of this model is scaled onto the Elo rating scale. This enables us to calculate the expected result of any rated learner solving exercises from any rated text.

Lexical frequency is known to be a powerful predictor of text readability, (Chen and Meurers, 2016). We use the normalized mean of the lexical frequencies of the tokens in a text as a feature. Additionally, we use mean token length and mean sentence length, as they are also used in classic readability measurements, (Kincaid et al., 1975; Flesch, 1979). These three features are scaled for a simple 3-variable regression model.

Currently, data for training this model are partitioned into two types: texts from sources with simplified language vs. texts from difficult sources. We label simple texts with a value of 0.2 and difficult texts with $0.8 .^{4}$ We aim for a model that produces a correct ordering of texts with respect to their difficulty. We do not need an exact estimate. If the learner Elo ratings based on these estimates correlate well with the ratings from the testing context, we consider this estimator accurate enough. In Section 5 we show that so far, this indeed seems to be the case.

The model outputs typically range between 0 and 1. We scale these values to Elo ratings according to formula 4 . This transformation is based on the Elo rating distribution acquired in the testing context. The bounds are clamped at $(0,1)$, as some texts may get a high difficulty value (sometimes even $>2$, in extreme cases).

$$
f(x)= \begin{cases}600, & \text { if } x<-0.4 \\ 1000 x+1000, & \text { if }-0.4 \leq x \leq 1.4 \\ 2400, & \text { if } x>1.4\end{cases}
$$

\section{Concept graph}

We are interested in finding a model for the "natural" order in which learners acquire linguistic concepts-directly from learner data. Learners will find some orders more natural than others-

\footnotetext{
${ }^{4}$ This is a simplification, which does not reflect reality accurately, as not all texts from a given source are of equal difficulty. However, simply fitting a low dimensional, high-bias estimator such as this yields a reasonable baseline model that generalizes well enough to other texts. We will explore more sophisticated models in the future.
}

e.g., when some concept is a requirement for another. The fact that one concept "precedes" another is called the surmise relation in Knowledge Space Theory (Doignon and Falmagne, 1999).

Often the domain model in ITS is built by eliciting domain knowledge from experts. We devised a baseline model to infer such relations from user data, without supervision. As we mentioned, each test question is mapped to a certain linguistic concept, and we store all test results from all students. Based on these results, we know to what extent which users have mastered which concepts; from this, we can try to tell apart the more basic concepts from the more advanced ones.

The aim is to build a partial order over the set of all concepts $C$, which specifices which concepts are related-i.e., we write $c_{2} \rightarrow c_{1}$ to mean concept $c_{2}$ presupposes (or implies) concept $c_{1} .5$

Given a set of users $U$, we build a matrix of "mastery" scores $M$, of dimension $|U| \times|C|$. Every element $M_{i j}$ is the proportion of correct answers that user $u_{i}$ has given for concept $c_{j}$. In the current implementation, we consider each "user" to be a single test session. If the same person completes the test at different times, they will be treated as different users for the purpose of computing consistency. This is done to take into account the fact that a user's level of proficiency changes over time.

For every pair $c_{1}, c_{2} \in C$, we check whether $c_{2} \rightarrow c_{1}, c_{1} \rightarrow c_{2}$ or $c_{1} \perp c_{2}$. We compare all columns of $M$ pairwise. Let $c_{j}$ denote column $j$ of $M$ and $c_{k}$ column $k$. To check whether $c_{k} \rightarrow c_{j}$ is true, we define a logical function $C O N_{u}$ which checks that user $u$ is consistent with this relation:

\begin{tabular}{c|c|c}
$M_{u k}$ & $M_{u j}$ & $\operatorname{CON}_{u}\left(c_{k} \rightarrow c_{j}\right)$ \\
\hline 0 & 0 & 1 \\
1 & 0 & 0 \\
0 & 1 & 1 \\
1 & 1 & 1
\end{tabular}

Again, here $c_{k}$ is the "harder" concept than $c_{j}$. Thus, if $M_{u k}=0$ (user $u$ knows nothing about concept $c_{k}$ ), that is consistent with $c_{k} \rightarrow c_{j}$ regardless of the value of $c_{j}$. Conversely if $M_{u j}=1$ (user $u$ mastered concept $c_{k}$ perfectly), that is consistent with $c_{k} \rightarrow c_{j}$ regardless of the value of $c_{k}$.

In practice, the values in $M$ are fractions between 0 and 1 . Therefore we introduce two thresh-

\footnotetext{
${ }^{5}$ We would like to say that $c_{1}$ is a prerequisite for $c_{2}$, but that may be too strong a claim. However we may be able to learn from the data that typically $c_{1}$ is mastered before $c_{2}$.
} 
old parameters to map $M_{i j}$ to zeroes and ones: we believe that a user $u$ really does not know the (harder) concept $c_{k}$ if $M_{u k} \leqslant \bar{\theta}_{\text {guess }}$ : the "guessing" upper bound, below which we believe that the user does not know the concept, while sometimes only guessing the correct answer. Analogously, we say that a user $u$ has mastered quite well the (easier) concept $c_{j}$ if $M_{u j} \geqslant \underline{\theta}_{\text {master }}$ : the "mastery" lower bound, above which we believe that the user knows the concept, while sometimes making a few mistakes.

To check whether $c_{k} \rightarrow c_{j}$, we compute the proportion $\sigma$ of all users who are consistent with this relation $c_{k} \rightarrow c_{j}$, as follows:

$$
\sigma\left(c_{k} \rightarrow c_{j}\right)=\frac{1}{n} \sum_{u \in U} \operatorname{CON}_{u}\left(M_{u k}, M_{u j}\right)
$$

where:

$$
\operatorname{CON}_{u}\left(M_{u k}, M_{u j}\right)= \begin{cases}1 & \text { if } M_{u k} \leqslant \bar{\theta}_{\text {guess }} \\ 1 & \text { if } M_{u j} \geqslant \underline{\theta}_{\text {master }} \\ 0 & \text { otherwise }\end{cases}
$$

$n$ is the total number of users. $\theta_{\text {guess }}$ and $\theta_{\text {master }}$ refer to the thresholds of guessing and mastering respectively. That is, we ignore all users where the level of proficiency in $c_{1}$ and $c_{2}$ suggests that user only partially understands both concepts, since they do not support to the consistency from $c_{k} \rightarrow c_{j}$.

We then apply a consistency threshold $\theta$ such that, if $\sigma\left(c_{2} \rightarrow c_{1}\right)>\theta$, we add the relation $c_{2} \rightarrow$ $c_{1}$ to our partial order.

Finally, we represent the partial order as a directed acyclic graph (DAG), where each path in the graph represents a possible prerequisite route toward learning a concept. For example, if we wish to obtain a complete syllabus for a language course, then we can find a total order compatible with our partial order (i.e., a linear extension) by topologically sorting the nodes in the graph.

We tested this approach with a set of over $620 \mathrm{~K}$ answers gathered from 700 users, and manually evaluated the results, setting $\theta=.7$.

Our domain experts confirm that the resulting graph provides a plausible model for the relations between the concepts in the language.

\section{Experiments}

\subsection{Elo burn-in}

In conventional Elo rating systems, the ratings of both actors are updated after each match. Our goal

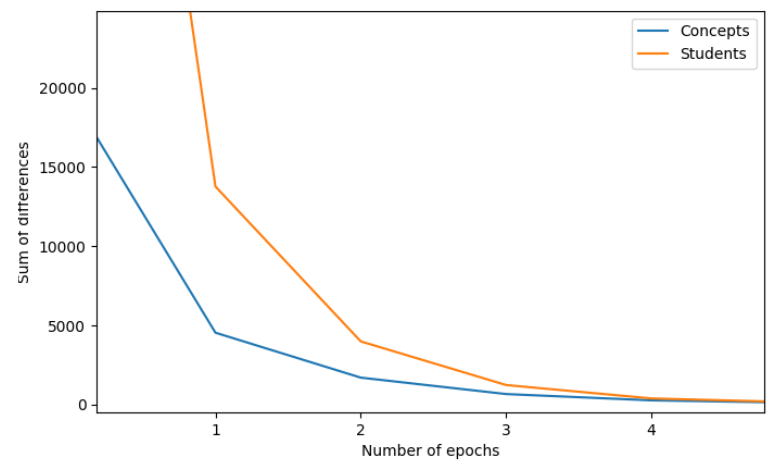

Figure 1: Learning concept Elo ratings with burn-in

is to learn a stable rating for each language concept, which we can keep unchanged as learners take further tests and improve. The rationale behind this is that the concepts don't become less difficult for everyone if a learner masters it.

To achieve this, we perform a burn-in simulation based on the user data we have collected. Specifically, we take the entire collection of data and calculate the Elo updates for each data pointuser $\mathrm{U}$ doing exercise $\mathrm{E}$-in chronological order. We do this for repeated epochs until the sum of differences between epochs nears zero. This is illustrated in Figure 1.

Once convergence is achieved, we reset the student ratings, and recalculate them from scratch with the fixed concept ratings. This yields a system where the learners' ratings are comparable and independent for any learner, including future ones.

\subsection{Test and exercise simulation}

To verify that one test (of 300 items) is sufficient for a learner to reach her current "true" rating, we performed simulation experiments. Concretely, we measured how many test items a new learner (e.g., with a rating of 1800 Elo) must answer in order to settle on her rating.

In this simulation, each student is initially rated as 1500 , and complete some test items. We use the Elo expectation formula to get the response accuracy for the simulated actors. We perform randomized simulations of actors performing one 300 -item test with this average response accuracy and observe the results, shown in Figure 2. The actors' responses-correct or false-are sampled randomly according to her supposed correct rate. We also add a small amount of normally distributed random noise. As Figure 2 shows, in this case, one 300-item test is enough to reach one's "true" rating. 


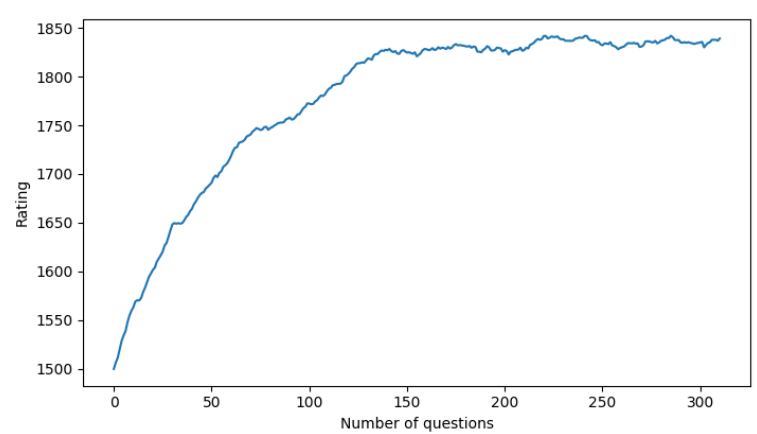

Figure 2: Simulation: test items

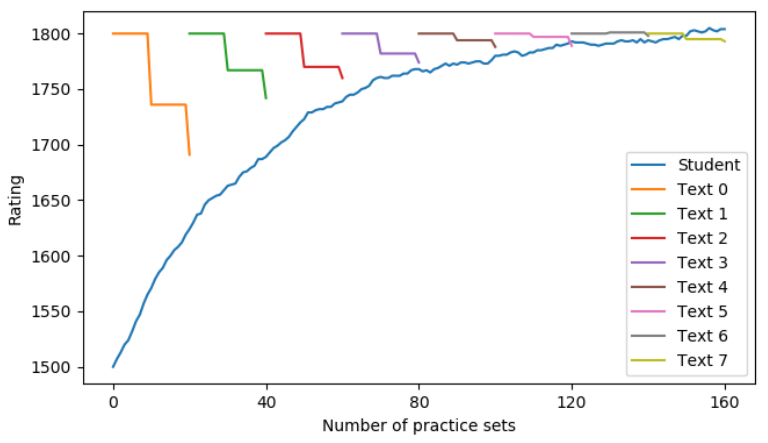

Figure 3: Simulation: exercises based on texts

In contrast to the test item context, in the context of text-based exercises, the Elo ratings will be updated over time both for for learners and practice texts. We conducted a simulation to visualize the rating trends.

In the exercise simulation, the simulated actor has the same starting point as in the simulation for tests-1500. We fix her rate of correct responses, e.g., as $50 \%$, and the actor is practicing only simulated texts rated at 1800 . This means that the theoretical upper bound of the actors' rating is, by definition, under $1800 .{ }^{6}$ The same normally distributed noise is introduced in this simulation as above. The data in our system shows that texts typically contain 10 snippets (exercise sets) or fewer. After a full pass through a text, namely, 10 problem sets, the ratings for the text are updated with respect to this particular actor. In this simulation each text is to be practiced twice. Figure 3 shows the result of this simulation. We stopped the simulation after 8 texts (each practiced twice) because the rating of the actor had converged to 1800. Since the student begins far below 1800 , the first several problem sets will have a substantial drop in their rating, due to the large initial dif-

\footnotetext{
${ }^{6} 50 \%$ success rate in a competition setting means the players are perfectly matched; since the text is rated 1800 , the learner's Elo rating should also reach 1800 and remain there.
}

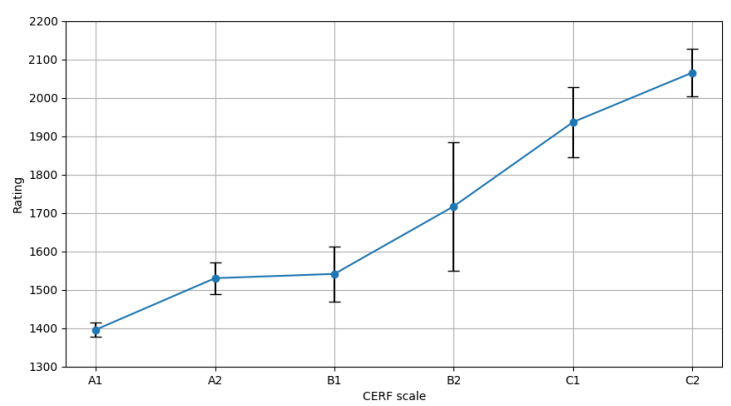

Figure 4: Expert annotated CEFR groups vs. mean Elo rating for each CEFR level

ference between student's rating and text's difficulty estimate. This initial burn-in process soon ends, after the student practice several snippets. As we can observe in Figure 3, the initial burnin process ends after 3 texts-10 snippets/practice sets per text, each text practiced twice, yielding 60 snippets - the subsequent ones showing only a slight change. The simulation is based on the assumption that the student will go though every problem set in strict order. In reality, this is not very likely. We can therefore infer that this student can reach a rating around 1800 rating after practicing with less than 140 snippets/problem sets.

\section{Results}

The result of primary interest is how well the Elo ratings given by our system correspond to CEFR levels assigned to the learners by the expert teachers, who estimate the learners' proficiency based on a wide range of assessment criteria, including written essays and oral exams. The relationship between Elo and CEFR is illustrated in Figure 4.

The figure shows the means and $95 \%$ confidence intervals of the Elo ratings for the CEFR levels of students, assigned subjectively by the teachers. The numbers of students at each CEFR level in our experiments are given in Table 1. The data in Figure 4 comes from 142 students whose CEFR levels were established independently of these tests by the teachers. Although language competency encompasses several skills-reading, writing, aural comprehension, speaking - and the learner may be at different levels in different skills, we normally expect that the competencies across different sills are fairly well correlated. The correlation om Figure 4 is 0.90 . Thus, the data in the figure indicate good correlation between our rating method and actual proficiency.

While assessing and modeling the improvement 


\begin{tabular}{|l|r|}
\hline CEFR & Students \\
\hline A1 & 48 \\
A2 & 31 \\
\hline B1 & 18 \\
B2 & 6 \\
\hline C1 & 17 \\
C2 & 22 \\
\hline Total & 142
\end{tabular}

Table 1: Number of CEFR rated students in Figure 4

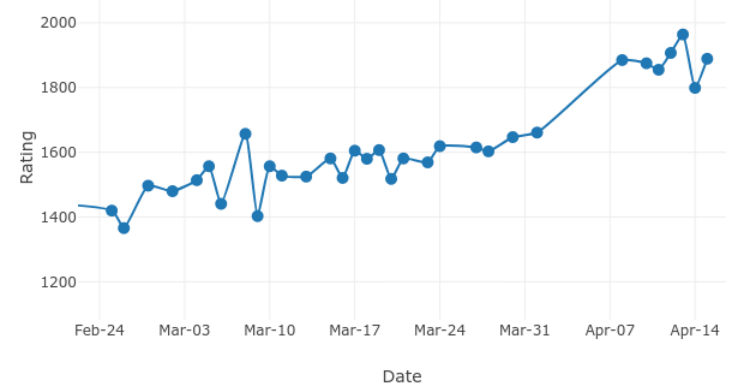

Figure 5: A representative student test Elo progress

of learners is largely in the realm of future work, we have interesting preliminary data of learners' improvement. Figure 5 shows an example of the progress of a typical actual learner taking tests. The learner takes (randomized) tests over a period of time, with the rating improving consistently. In this example, we see an increase of almost 300 in average Elo during the periods. The improvement is attributed to the fact that the learner has covered new material during the period, mastered more of the concepts in the test, and consequently scoring better on those questions.

Another crucial question is how well our Elo estimates from the exercise context correlate with Elo ratings from the testing context. For this we also have initial results, shown in Figure 6. We have so far collected only a modest amount of learner data, and therefore the conclusions drawn from the data are preliminary. Investigating this correlation requires substantial data from learners who have worked in both contexts: practicing with exercises based on texts, and completing tests. The figure shows results for the top 17 students, who have completed at least 1000 exercise sets (text snippets, in orange) and at least 300 test items (in blue)—sorted according to their test rating. The figure shows good correlation between the two rat-

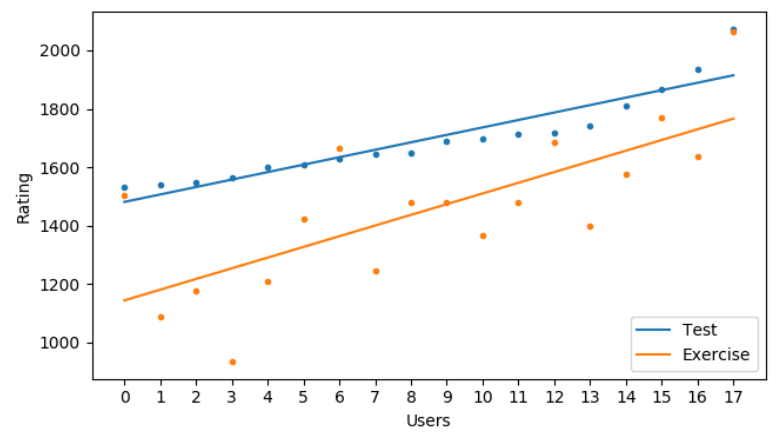

Figure 6: Correlation between test and text-based exercise ratings $(\rho=0.79)$

ings for the students. At present, the correlation between test and practice Elo score is 0.79 .

The last goal in our work is to investigate the relationship between the concept graph and the Elo ratings. Figure 7 shows a small sub-graph of the concept graph. The complete concept graph 8 can found in the Appendix. From Figures 7 and 8, we can see that typically (though not always), if concept A surmises concept B, A will have a higher Elo rating than $\mathrm{B}$. This makes sense, as more difficult concepts should surmise easier ones. The Elo ratings and the graph structure do not correspond perfectly. Expecting that real-valued linear ratings can accurately describe the natural order of concepts is unreasonable. Since the graph and the Elo ratings describe different processes, it is not surprising to find inversions in the graph, such as between concepts 98 and 93, in Figure 7. The structure of the graph, its relation to the difficulty of concepts, and the natural learning order of concepts is an key future research topic.

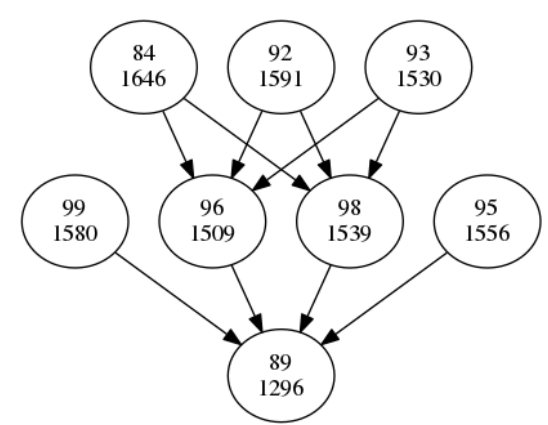

Figure 7: Sub-graph of the complete concept graph (see Supplementary Materials). Arrows denote implication. Top number: ID of the concept (appendix A); bottom number: Elo score of the concept (appendix B). 


\section{Current problems}

We are in the process of collecting user data and evaluating the methods of assessment presented in this paper. Subjective CEFR ratings are being collected from the teachers for some of the students. While the number of hand-labeled CEFR ratings is modest at the time of writing, it is sufficient to indicate that using Elo-based ratings for measuring language proficiency shows promise. Building domain models based on the concept precedence graph is another direction of research.

Comparing our ratings and expert-annotated proficiency levels in larger quantities will raise the confidence of our method. We must note that a single value cannot be expected to describe the language proficiency of a learner completely, as there are several aspects of the language to master.

We plan to compare other Elo-based models, such as Glicko (Glickman, 1999), and TrueSkill (Herbrich et al., 2007), with our Elo rating formula. Robust methods for obtaining numerical estimates of skills enables us to develop ICALL systems, by facilitating the quantitative evaluation of skills, and the resulting improvement of the learners.

\section{Conclusions}

The main contributions of this paper are:

- We adapt and evaluate Elo-based rating formulas for modeling language learners' proficiency, as well as the difficulty of texts and linguistic concepts, not only the difficulty of questions/test items.

- We obtain static difficulty ratings for the linguistic concepts by performing an initial burn-in for the Elo ratings based on a large amount of learner data, and then assess students' proficiency using the learned Elo ratings. Feedback from language teachers/experts indicates that the ratings correlate with their estimates of learner competency.

- We use a linear-regression model of text difficulty as an estimator, to obtain Elo ratings for the texts. This enables us to rate the performance of learners, who practice with exercises generated from the texts. Preliminary results indicate that there is a positive correlation between ratings in the exercise and the test contexts.
- We build a partial order over all concepts found in the domain, and visualize the partial order as a DAG over concepts. The concept graph is not linear as the Elo ratings. The structure of the concept graph and the Elo ratings of the concepts generally agree in that the graph displays a strong tendency of decreasing rating from the more complex concepts to the more fundamental concepts, as indicated by the actual data collected from the learning process.

In sum, the proposed methods enable us to rate the proficiency of the current and future language learners, which is a fundamental goal in ITS and ICALL.

\section{Acknowledgements}

Work was supported in part by the Academy of Finland, Project FinUgRevita, Grant No. 267097, and by HIIT-Helsinki Institute for Information Technology.

\section{References}

Ivon Arroyo, Beverly Park Woolf, Winslow Burelson, Kasia Muldner, Dovan Rai, and Minghui Tai. 2014. A multimedia adaptive tutoring system for mathematics that addresses cognition, metacognition and affect. International Journal of Artificial Intelligence in Education, 24(4):387-426.

Hao Cen, Kenneth Koedinger, and Brian Junker. 2006. Learning factors analysis - a general method for cognitive model evaluation and improvement. In Intelligent Tutoring Systems, pages 164-175, Berlin, Heidelberg. Springer Berlin Heidelberg.

Xiaobin Chen and Detmar Meurers. 2016. Characterizing text difficulty with word frequencies. In Proceedings of the 11th Workshop on Innovative Use of NLP for Building Educational Applications, pages 84-94.

Albert T Corbett and John R Anderson. 1994. Knowledge tracing: Modeling the acquisition of procedural knowledge. User modeling and user-adapted interaction, 4(4):253-278.

Jean-Paul Doignon and Jean-Claude Falmagne. 1999. Knowledge Spaces.

William Dubay. 2009. Unlocking language: The classic readability studies. Professional Communication, IEEE Transactions on, 51:416 - 417.

Arpad E. Elo. 1978. The rating of chessplayers, past and present. Arco Pub., New York. 
Susan E Embretson and Steven P Reise. 2013. Item response theory. Psychology Press.

Rudolf Flesch. 1979. How to write plain English: Let's start with the formula. University of Canterbury.

Mark E Glickman. 1999. Parameter estimation in large dynamic paired comparison experiments. Journal of the Royal Statistical Society: Series C (Applied Statistics), 48(3):377-394.

Michael Heilman, Kevyn Collins-Thompson, and Maxine Eskenazi. 2008. An analysis of statistical models and features for reading difficulty prediction. In Proceedings of the third workshop on innovative use of NLP for building educational applications, pages 71-79. Association for Computational Linguistics.

Ralf Herbrich, Tom Minka, and Thore Graepel. 2007. Trueskill $^{\mathrm{TM}}$ : A Bayesian skill rating system. In B. Schölkopf, J. C. Platt, and T. Hoffman, editors, Advances in Neural Information Processing Systems 19, pages 569-576. MIT Press.

Anisia Katinskaia, Javad Nouri, and Roman Yangarber. 2018. Revita: a language-learning platform at the intersection of ITS and CALL. In Proceedings of LREC: 11th International Conference on Language Resources and Evaluation, Miyazaki, Japan.

J Peter Kincaid, Robert P Fishburne Jr, Richard L Rogers, and Brad S Chissom. 1975. Derivation of new readability formulas (automated readability index, fog count and Flesch reading ease formula) for Navy enlisted personnel.

Sharon Klinkenberg, Marthe Straatemeier, and Han LJ van der Maas. 2011. Computer adaptive practice of maths ability using a new item response model for on-the-fly ability and difficulty estimation. Computers \& Education, 57(2):1813-1824.

Mikhail Kopotev. 2010. Система прогрессивного тестирования Karttu (описание и первые результаты). Русский язык за рубежом, (3):23-29.

Mikhail Kopotev. 2012. Karttu: результаты языкового тестирования в школе и вузе. Формирование и оценка коммуникативной компетенции билингвов в процессе двуязычного образования: Сб. ст./Под ред. ЕЕ Юркова и др. СПб.: МИРС, pages 312-339.

David Little. 2007. The common European framework of reference for languages: Perspectives on the making of supranational language education policy. The Modern Language Journal, 91(4):645-655.

Philip I Pavlik Jr, Hao Cen, and Kenneth R Koedinger. 2009. Performance factors analysis-a new alternative to knowledge tracing. Online Submission.
Radek Pelánek. 2016. Applications of the Elo rating system in adaptive educational systems. Computers \& Education, 98:169-179.

Steven Ritter, John R. Anderson, Kenneth R. Koedinger, and Albert Corbett. 2007. Cognitive tutor: Applied research in mathematics education. Psychonomic Bulletin \& Review, 14(2):249-255. 


\section{A List of concepts}

\begin{tabular}{|c|c|c|}
\hline ID & Rating & Name of concept \\
\hline 6 & 1602 & Lexicology. Lexical semantics \\
\hline 7 & 1860 & Collocations \\
\hline 8 & 1679 & Lexicology. Coordination of words \\
\hline 9 & 1382 & Verb. Case government \\
\hline 10 & 1541 & Verb. Prepositional government \\
\hline 11 & 1550 & Adjectives \\
\hline 12 & 1509 & Nouns \\
\hline 19 & 1471 & Impersonal verbs (except verba meteorologica) and their government \\
\hline 20 & 1482 & Predicative adverbs and their government. Existence, state, time \\
\hline 21 & 1480 & Predicative adverbs and their government. Necessity, possibility, impossibility \\
\hline 22 & 1577 & Negative constructions with predicative 'be' (and synonyms), genitive of negation \\
\hline 23 & 1423 & Sentences with dative subject: 'Кате 25 лет' \\
\hline 24 & 1445 & Expressions of time, place and manner. Preposition-free expressions \\
\hline 25 & 1621 & Constructions with cardinal numerals \\
\hline 26 & 1470 & Constructions with collective numerals \\
\hline 27 & 1652 & Genitive plural of Pluralia tantum words \\
\hline 28 & 1630 & I declension. Tyре 'музей-музеи, воробей-воробьи' \\
\hline 29 & 1595 & I declension. Tyре 'санаторий' \\
\hline 30 & 1662 & I declension. Fleeting vowels and alternations я, e, ё / й ('заяц-зайца, заём-займа') \\
\hline 31 & 1608 & I declension. Type 'карандаш' \\
\hline 32 & 1671 & I declension. Tyре 'адрес-адреса' \\
\hline 33 & 1562 & I declension. Type 'солдат-много солдат, сапог-пара сапог' \\
\hline 34 & 1586 & I declension. Туре '-анин/-янин, -ин' \\
\hline 35 & 1518 & I declension. Tyре 'дерево-деревья’ \\
\hline 36 & 1616 & II declension. Tyре 'армия' \\
\hline 37 & 1603 & II declension. Tyре на -ня \\
\hline 38 & 1713 & II declension. Tyре 'статья' \\
\hline 39 & 1616 & Fleeting vowel in genitive plural \\
\hline 40 & 1504 & Nouns with prepositions $\mathrm{B} / \mathrm{Ha}$ ending in $-\mathrm{y} /-$-ю in prepositional singular \\
\hline 41 & 1619 & Nouns ending in $-\mathrm{y} /-\mathrm{ю}$ in prepositional singular and $-\mathrm{a}$ in nominative plural \\
\hline 42 & 1644 & Possessive adjectives. Туре 'лисий' \\
\hline 43 & 1601 & Ordinal numbers. Tyре 'третий' \\
\hline 44 & 1612 & Possessive adjectives. Туре 'мамин' \\
\hline 45 & 1543 & Cardinal numbers. 'Сто' vs. 'пятьсот, шестьсот, семьсот, девятьсот' \\
\hline 46 & 1608 & Quantifiers. Collective quantifiers in oblique cases \\
\hline 47 & 1528 & Quantifiers. Collective quantifiers 'оба, обе' \\
\hline 48 & 1594 & I conjugation. Tyре 'плакать' \\
\hline 49 & 1511 & I conjugation. Type 'рисовать' \\
\hline 51 & 1560 & II conjugation. Type 'молчать' \\
\hline 52 & 1674 & Preterite. Туре 'исчезнуть' \\
\hline 54 & 1570 & Regular verbs with vowel alternation \\
\hline 55 & 1512 & Resultative \\
\hline 56 & 1553 & Iterative / potential iterative / qualities \\
\hline 57 & 1507 & Expression of duration - 'за какое время' \\
\hline 58 & 1416 & Factual meaning of verbs \\
\hline 59 & 1817 & Aspect, expression of action completed in the past \\
\hline 60 & 1648 & Aspect, expression of capability/incapability. ('Тебе этого не понимать/понять!') \\
\hline 61 & 1509 & Inception of action \\
\hline 62 & 1525 & 'Забыть, успеть, удаться' + infinitive \\
\hline 63 & 1539 & 'Уметь, нравиться, любить' etc. + infinitive \\
\hline 64 & 1651 & 'Пора, ckopee' + infinitive \\
\hline 65 & 1553 & 'Нельзя, невозможно, не могу' + infinitive \\
\hline 66 & 1499 & 'He' + infinitive \\
\hline 67 & 1501 & Negative sentences \\
\hline 68 & 1411 & Imperative \\
\hline 69 & 1559 & Proximal future \\
\hline 70 & 1674 & Impersonal sentences. Infinitival sentences. Subordinate sentences ('если / прежде чем' + infinitive) \\
\hline 71 & 946 & Impersonal sentences. Infinitival sentences. Modal expressions \\
\hline 72 & 1580 & Impersonal sentences. Infinitival sentences. Sentences, with negative pronouns and adverbs \\
\hline 73 & 1701 & Generis-personal sentences \\
\hline 75 & 1516 & Passive and its relation to indefinite-personal sentences (equivalent of Finnish impersonal passive) \\
\hline 76 & 1800 & Stress: forms of verbal preterite \\
\hline 77 & 1706 & Stress: short adjectives \\
\hline 78 & 1736 & Stress: participles \\
\hline 79 & 1548 & Nouns. Type A: stress on the stem \\
\hline 80 & 1719 & Nouns. Type B, B1, B2: stress on the ending \\
\hline
\end{tabular}


1633 Nouns. Type C, C1: stress on the stem in singular, on the ending in plural

1614 Nouns. Type D, D1: stress on the ending in singular, on the stem in plural

1610 Nouns. Tyре 'нож-ножом, сторож-сторожем'

1646 Declension of adjectives. Tyре 'хорошего'

1295 Place of adverbs of time, place and manner

1693 Place of negation in the sentence

1785 Place of participles in the sentence

1618 Place of gerunds in the sentence

1296 Place of pronouns in a phrase

1589 Word order in sentences introducing direct quotations

1681 Second person singular imperative in conditionals ('если')

1591 Usage of 'сам' and 'один'

1530 Sentences of type 'Знаю его как врача.'

1567 Sentences of type 'Быть грозе'

1556 Sentences of type 'Лодку унесло ветром'

1509 Genitive plural

1537 Frequent prefixed verbs of motion + prepositional constructions

1539 Animate noun object

1580 Unprefixed verbs of motion

1121 Unstressed fleeting vowels in roots and suffixes of nouns and adjectives

1391 Unstressed vowels in verbal forms

907 Unstressed vowels in roots

1452 Unstressed vowels in case endings

1179 Unstressed vowels in prefixes

370 Unstressed vowels in suffixes

1557 Unstressed vowels linking compounds

1224 Unstressed particles не and ни

357 Letter $\Gamma$ in ending -ого (-его)

1443 Letter й

1241 Letter $ч$ and $ш$ before $\mathrm{H}$ and $\mathrm{T}$

1427 Letters $\mathrm{b}$ and $\mathrm{b}$ :

1500 Vowels in verbal endings

1451 Vowels in the infinitive (indefinite form) before -ть

1365 Vowels not after sibilants and ц

1231 Vowels after sibilants and ц

1091 Voiceless and voiced consonants

1378 Consonant clusters at the juncture of morphemes

1654 Double and single $-\mathrm{H}$ - in suffixes of adjectives and nouns

2025 Double and single $-\mathrm{H}$ - in suffixes of full and short forms of adjectives

1706 Double and single $-\mathrm{H}$ - in suffixes of past passive participles and corresponding adjectives

1414 Double and single $-\mathrm{H}$ - in words derived from adjectives and participles

1341 Double consonants in borrowed roots and suffixes

1529 Double consonants in native roots

1131 Double consonants at morpheme juncture

997 Silent consonants

1050 Peculiarities of spelling of certain roots

1273 Peculiarities of spelling of certain suffixes

1868 Joint vs. separate spelling of negation 'He': verb (+participles)

1320 Joint vs. separate spelling of negation ' $\mathrm{He}$ ': pronoun

1296 Joint vs. separate spelling of negation 'не': adverb (+'несколько')

1475 Joint vs. separate spelling of negation ' $\mathrm{He}$ ': adjectives (+full-short)

1490 Joint vs. separate spelling of negation ' $\mathrm{не}$ ': noun

1563 Joint vs. hyphenated spelling: adjectives

1590 Joint vs. hyphenated spelling: numerals

1059 Joint vs. hyphenated spelling: pronouns

1312 Joint vs. hyphenated spelling: adverbs

1555 Joint vs. hyphenated spelling: common nouns

1484 Joint vs. hyphenated spelling: function words

1836 Joint vs. hyphenated spelling: proper names

1555 Capitalized vs. lowercase: astronomical/geographical names

1664 Capitalized vs. lowercase: posts, titles, awards

1738 Capitalized vs. lowercase: names of official organizations

1898 Capitalized vs. lowercase: names linked to religion, historical epochs and events

1718 Capitalized vs. lowercase: names of trademarks, documents, works of art

1309 Capitalized vs. lowercase: proper names of persons, animals

1527 Accusative/ergative subject + Impersonal verb: 'Васю тошнит'

1467 Prep+genitive subject + Impersonal verb: 'У меня болит голова/шумит в голове'

1496 Dative subject + adverb: 'мне (стало) плохо/нужно/скучно'

1548 Dative subject + impersonal verb: 'мне идет/надоело/везет/хватит'

1431 Dative subject + impersonal-reflexive verb: 'мне нравится/кажется/пришлось' 
B Concept graph 


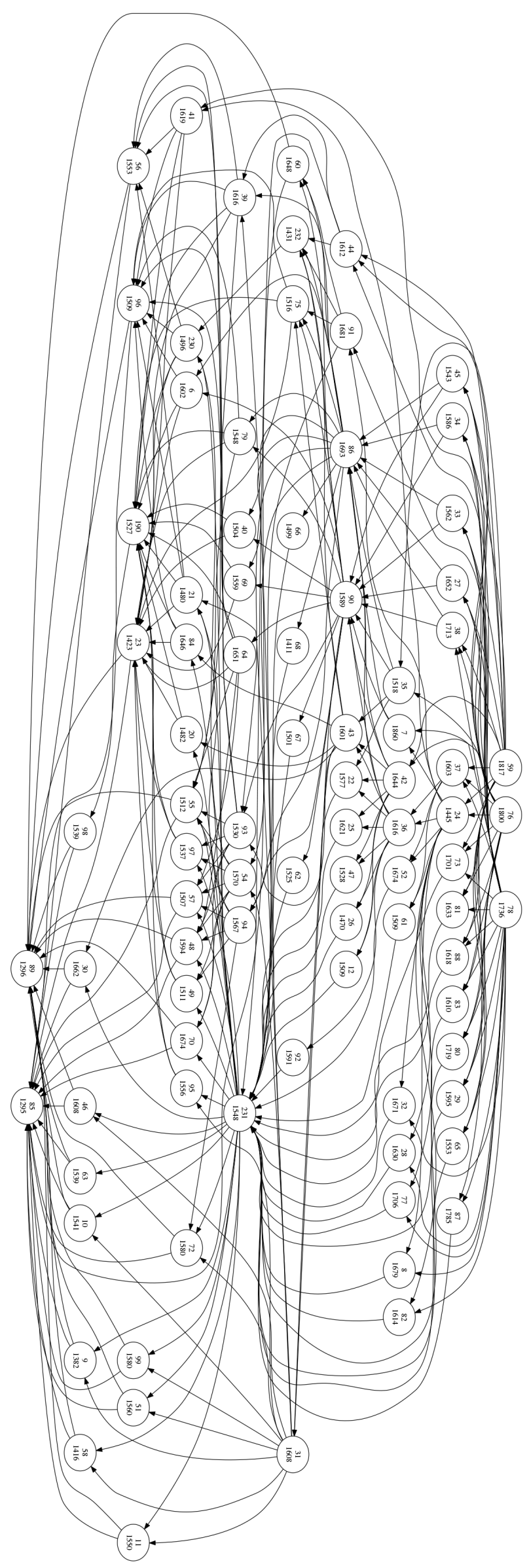

Figure 8: The full concept graph. 